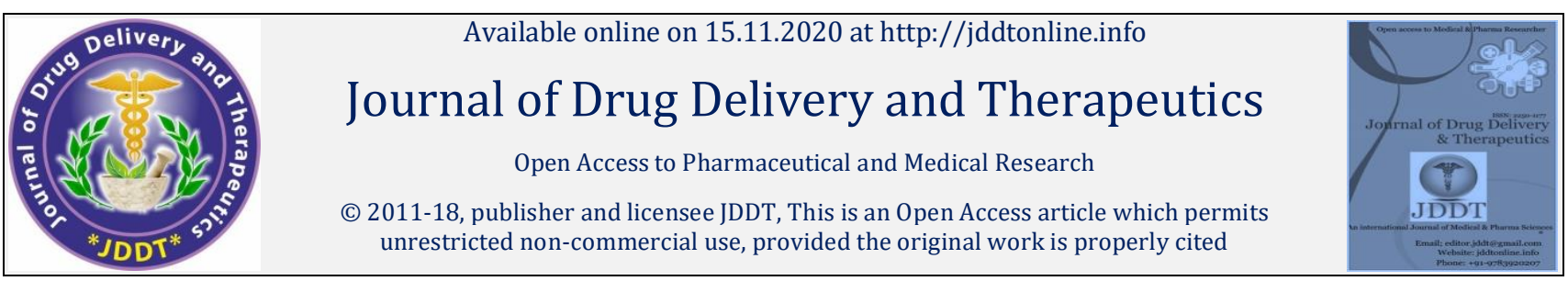

Open $\odot$ Access

\title{
Biosimilar Biologic Drugs: A Systematic Approach to Development, Manufacturing and Clinical Applications
}

Kaiser Jay Aziz-Andersen, MB, MS, PhD, FACB, FACS

Director, Grand Medical Institute, Bowie, Maryland, USA

\begin{abstract}
This publication addresses biosimilar biologic drugs development and future innovations. Emphasis is placed on quality system approaches to the development and availability of new biosimilar drug products presented in premarket applications. For approvals of new biosimilars, the sponsors of premarket applications must present analytical and biological characterization to demonstrate that a proposed biosimilar drug is highly similar to the licensed reference product. The premarket application protocol requires a sponsor to describe the biosimilar product's PK/PD clinical data comparing its safety, efficacy, and immunogenicity to that of the licensed reference product. Emphasis is placed on Quality by Design (QbD), Validation, Verification, and c-GMP risk-based monitoring criteria. A brief discussion is presented on risk-benefit assessment that guides the clinical use of the new biosimilar drug product by providing patients organized data and appropriate labeling information in conformance with the new biosimilar drug's intended clinical use.
\end{abstract}

Keywords: biosimilar biologic drugs, safety, efficacy and immunogenicity, Quality by Design (QbD)

Article Info: Received 19 Sep 2020; $\quad$ Review Completed 13 Oct 2020; $\quad$ Accepted 21 Oct 2020; $\quad$ Available online 15 Nov 2020

Cite this article as:

Aziz-Andersen KJ, Biosimilar Biologic Drugs: A Systematic Approach to Development, Manufacturing and Clinical

Applications, Journal of Drug Delivery and Therapeutics. 2020; 10(6):191-194

http://dx.doi.org/10.22270/jddt.v10i6.4373

*Address for Correspondence:

Kaiser Jay Aziz-Andersen, MB, MS, PhD, FACB, FACS, Director, Grand Medical Institute, Bowie, Maryland, USA

\section{Introduction:}

The rising cost of biologic medicines has necessitated the development of biosimilar drugs. The US Drug Price Competition and Patent Term Restoration Act of 1984, widely known as the Hatch-Waxman Act, introduced biosimilar drugs legislation to establish a regulatory pathway for pharmaceutical companies to develop identical and more cost-effective copies of drugs that were facing patent expiration. These research and development (R \& D) activities lead into production of novel biological products derived from animal, human, or microorganism sources. These types of products include protein-based enzymes, hormones, monoclonal antibodies, vaccines, blood products, gene and cellular therapeutics. These types of products are expected to decrease health care costs at the same time having the benefits of established generic drugs. They must also contain a version of the active substance of the innovator reference product (previously approved marketed biologics). These types of products known as biosimilar medicines must contain similar quality characteristics, bioactivity, safety and efficacy profiles in comparison to the innovator reference product. The emphasis is placed on quality aspects of the biosimilar product going through all the necessary production steps including design controls, process controls, formulation, vialing, freeze drying, storage, and packaging. The FDA defines biosimilar products to mean that the biosimilar product is highly similar to the reference innovator product not showing any significant differences in terms of safety, potency and efficacy. ${ }^{1}$

Biosimilar medicines are highly similar to FDA approved biologic drugs. The sponsors of premarket applications clearly define their product's intended clinical use playing an important role for use of biosimilar medicines in specialty therapy categories such as immunology, endocrinology, and oncology. The new biosimilar products approved by FDA play a pivotal role in the clinical treatments of patients suffering from life threatening diseases such as cardiac myopathies, carcinoma, sarcoma, and lymphoma. The US biosimilar approval process requires a thorough characterization of the new biosimilars with clinically meaningful outcomes. Sponsors of new biosimilars follow the appropriate International Council for Harmonization (ICH) guidelines in regard to clinical PK/PD requirements, safety, and efficacy studies. The FDA guidances for extrapolation and interchangeability state that data derived from clinical studies should be adequate to demonstrate purity, potency, safety, and the intended clinical use of the new biosimilar in comparison to previously approved 
licensed biologics. This publication emphasizes FDA's quality system approach to design of studies for clinical applications for designated specialty therapy categories. In this publication, emphasis is placed on a stepwise approach to include analytical studies, biological characterization, preclinical and clinical testing of the proposed biosimilar product presented in the premarket applications. The sponsors of premarket applications must present analytical characterization methodology in determining pharmacokinetic (PK), pharmacodynamic (PD) and comparative clinical studies to demonstrate that the proposed biosimilar product is highly similar to a licensed biologic reference product. Discussions are presented on Quality Systems approach to current Good Manufacturing Practices (c-GMP's) for biosimilar applications (Design Controls, Validation and Verification studies, Good Manufacturing practices and effective total product life-cycle CMC strategy). Discussions are included on clinical aspects of the new biosimilar product's specific requirements (i.e., Immunogenicity Assessment, Extrapolations \& Interchangeability in conformance with specific recommendations described in the FDA's guidance on biosimilar labeling). Emphasis is placed on the Quality Risk Management approach to design of studies by providing oversight and objective review of risk-benefit analysis that guides the clinical use of the new biosimilar drug product by providing patients organized data and appropriate labeling information in support of the new biosimilar's intended clinical use. ${ }^{2}$

\section{Manufacturing Developments and Challenges:}

Continuous quality improvement (CQI) and continuous quality monitoring (CQM) is gaining attention in FDAapproved c-GMP certified facilities. Traditionally, pharmaceutical manufacturing drug dosage forms are put together in a systematic step-by-step assembly of various components known as batch manufacturing process. In a batch process, each sequential step is completed before moving on to the next step; and similarly, one batch must be finished before starting another. Pharmaceutical-biological batch processing requires multiple pieces of complicated equipment and machinery, the raw material and intermediates may need to be stored and moved from one site to another to complete the manufacturing process. ${ }^{3,4}$

Application of CQI and CQM techniques provide a resource saving to facilitate production of the biosimilar drug products with less total equipment down time. Hazard Analysis and Critical Control Point (HACCP) system is a risk management tool that, when implemented properly, can also be used as an internal inspection tool. However, for the HACCP approach to work effectively, auditors, investigators and manufacturers must have training and protocols to develop a HACCP plan. Responsible individuals need to be trained in developing and modifying the HACCP plan and reviewing records. HACCP approach provides quality by design $(\mathrm{QbD})$ techniques for biosimilar products that relate to safety and risk management system and can be integrated into manufacturing operations. HACCP is a preventive system of monitoring hazard/risk control rather than a reactive one. Pharmaceutical/Biosimilar manufacturers can use it to ensure safer products for consumers. In order to help ensure safer products, the HACCP system can be designed to guide the identification of hazards, establish controls, and monitor these controls. A hazard can be any condition that results in an adverse consequence(s) detrimental to the product, patient, or health care professional and includes the environment. Application of HACCP principles deals with safety and hazards associated with a product or process. An example of this may be that a biosimilar manufacturing system may require a weekly review and/or approval of production records. Another example may be a regulatory requirement that each lot of incoming raw material be tested; however, under a HACCP system only periodic testing and verification may be sufficient to assure the safety of the material.5,6 Ideally in CQI-CQM approaches, the biosimilar materials move nonstop within the same manufacturing facility, eliminating any hold time between steps. Because under CQI and CQM approaches a small downtime is involved, the risk of human error under the HACCP umbrella is significantly reduced. Thus the operating cost is also reduced as less product handling is required during the process. $5,6,7$

\section{Biosimilar Drug Manufacturing CMC Strategy:}

Biosimilar drug development and Manufacturing section of premarket application includes The Chemistry, Manufacturing and Controls (CMC) aspects of FDA's regulations. These applications contain the biosimilar drug's stereo-chemical properties, its manufacturing process development, and production control mechanisms dealing with analytical testing both in-process and finished drug product.1,3,5 The challenge for a biosimilar sponsor is to have a manufacturing process with adequate controls to ensure that the proposed biosimilar product closely matches the reference drug product and demonstrates its biosimilarity in clinical applications.

The very first step for biosimilar CMC strategy is to establish the basic structural properties similar to the reference innovative drug product. Generally, structural information is available through innovative drug products' referenced publications. For instance, information such as the amino acid sequence or scientific category of the reference product becomes part of the CMC strategy (ie., IgG antibody, enzymes, fusion proteins, formulation, mechanism of action, dosage, etc.). The backbone connection of biosimilar types of products are expressed as Critical Quality Attributes (CQAs) that must closely match the new biosimilar product to that of the reference product. In some situations, CQAs may represent the protein functional aspects of the product, such as an antibody binding to its target receptor or enzyme activity output, which can be expressed in the form of mechanism of action (MOA). Whereas other situations may result in structural modifications such as glycosylation, which can result in effector functions such as pharmacokinetic and pharmacodynamics profiles. Some CQAs may represent molecular aggregation or degradation which can impact biological activity outcomes. For instance, biosimilar product's structural degradation may affect therapeutic immunogenicity.,2,5 Additionally, it may be necessary to evaluate the manufacturing process related residual impurities such as host-cell proteins (HCP) and host-cell DNA (HCDNA). HCP related impurities are generally tested by immunoassays such as ELISA and HCDNA related impurities by polymerase chain reaction (PCR). These type of tests contribute to biosimilar products' safety profiles. $1,5,7$

\section{QbD Considerations for Biosimilars:}

The International Conference on Harmonization (ICH) defines QbD as a systematic approach to development that starts with predefined objectives of product concepts related to manufacturing process controls and quality risk management. The goal is to ensure that quality is established in the product from its inception to looking beyond the individual processes that are involved in the manufacturing of a biosimilar drug product and its intended user. QbD includes preclinical and clinical studies continuing through 
manufacturing and commercialization as part of the product lifecycle. Pharmaceutical QbD elements are based on scientific approach and reflect the degree of risks associated with changes to a product or process. These elements include the quality target criteria to provide quality target product profile (QTPP), critical quality attributes (CQAs), the performance parameters of risk assessments connecting critical material attributes (CMAs), and process critical control points (CCPs). The compilation of all of these elements leads to the concept of design space for the process and, by defining a particular biosimilar product's design space, it becomes easier to detect any modification or a major change in the final product.5,6,7,9

ICH Q8 (ICH 2009) describes the design space as the relationship between process inputs, which include both the attributes of material inputs as well as the process parameters, and critical quality attributes. The design space includes an evaluation of the potential variables within the manufacturing process to determine the impact of variation of those parameters on the biosimilar product quality. The design space defines ranges around each of those variables such that, as long as the process is operated within those ranges, consistent product quality is maintained. However, a more sophisticated Design of Experiments (DOE) approach or other statistically derived experimental design of a particular biosimilar drug product may be required. It is important to note that ICH Q8 addresses process evaluation; however, the design space requires a clear definition of analytical methodology and its capability. Methods that are not capable of detecting critical control points (HACCPbased CCPs) may not provide adequate information in regard to critical deviations for the biosimilar product. There are two major components of biosimilar product's design space--the product design and the process design. The product design describes the essential parameters that were determined as part of the product definition (i.e., physical, chemical and biological attributes of the biosimilar drug product as described in the QTPP). The product design should also include the criteria for the acceptable variability limits for product quality and stability. 1,4,5,10

\section{Biosimilar Risk Management:}

Biosimilar Drug product's risk management process begins with the systematic identification of known and foreseeable hazards based on the knowledge and experience of the reference product's information available in published literature. It is based on a systematic and informed decision providing greater insight into patient safety risks, fostering quality by design, and continuous improvement that leads to probability and predictability of the candidate biosimilar product's projected results. This includes not only the analytical performance characteristics, but also any foreseeable effects associated with the intended use of the biosimilar drug product. Any failures to meet explicit and implied claims are also evaluated as potential hazards. The main hazard would be to intended patients leading to a dangerous misdiagnosis or treatment. Another hazard might be a harmful delay in treatment. Following are the candidate biosimilar's life-cycle risk management elements that need to be addressed in premarket applications:

\section{- Hazard Identification}

- Risk analysis

- Risk evaluation

- Quality Control

- $\quad$ Production and post-production data monitoring
Quality risk management (QRM) is a critical component of an effective quality system framework of biosimilars. It can help guide the setting of specifications and process parameters for biosimilar drug manufacturing in assessing and mitigating the hazardous situation for the patient, who may be harmed by inappropriate treatment. Once a list of potential hazards is constructed, possible harm scenarios can be identified. In a typical risk analysis, each hazard is analyzed to estimate the probability that it will occur in an actual situation. The purpose of a preliminary hazard analysis is to identify the risks early so they can be systematically eliminated during the design phase of the biosimilar product or at least reduced to an acceptable level during manufacturing process controls. Risk management decisions are based on probability of harm to the intended user. 5,11

The individual biosimilar sponsor must decide the acceptable level of risk weighing the benefits to the patients and taking into account the current values and the needs of particular patient populations. There are five basic risk elements providing the linkage between biosimilar drug's material attributes and process parameters. These include hazard identification, risk analysis, risk evaluation, continuous quality control, and production monitoring Hazard identification includes the process of evaluating the manufacturing processes or product, or potential design control changes to that process/product in order to determine the hazards that may impact the final biosimilar product's intended user. In the hazard identification process the sponsor should take into account historical trends and/or information available through literature, as well as subject matter expert experience and recommendations. The goal at this stage is to select all possible risks and consequences. Risk analysis follows the identification of risks in terms of qualitative or quantitative values to the risk from the perspectives of likelihood of occurrence and severity of impact. In some situations, the ability to detect the hazard is part of the risk evaluation and scoring process, as part of batch rejection and therefore offers additional preventive protection to consumers. Risk evaluation is the summary of the information determined in the hazard/risk identification and risk assessment steps. This includes the process quality control steps described in the HAACP plan. The initial risk assessment exercise may result in risk levels as low, medium or high--low level being non-critical and high level being critical.5,6 HACCP built-in monitoring systems include risk control measures. The need for monitoring would be identified during risk analysis when the biosimilar product is being designed, where potential failure modes are identified through a Failure Modes and Effects Analysis (FMEA). Risk management according to ISO 14971 is a product of "total product life-cycle"(TPLC) process, which means it continues from design control to product being produced and is still in active use. During the biosimilar product life cycle, sponsors look out for the occurrence of previously unidentified hazards or indications or detection of residual risks. This is known as "acceptable safety information" in ISO 14971, which includes instructions for use, warnings and precautions, contraindications and limitations, performance characteristics and quality control aspects of the biosimilar product's clinical use. Additionally, external sources of user feedback, such as performance evaluations, customer complaints and adverse event reports, internal data sources are also monitored. 5,7

\section{Quality Assurance Strategy (QAS):}

The candidate biosimilar product's design controls play a key role in establishing HACCP-based critical quality 
attributes and manufacturing critical control points (CCPs) for quality monitoring purposes. Once this initial phase is established, it is necessary to implement the candidate biosimilar's design space, thereby facilitating consistency in the product outcome. The essential aspects of the QAS include, but are not limited to, raw material specifications, in-process controls, intermediate product testing protocols, and finished product release testing. The QA strategy is based on ICH set of controls, derived from current biosimilar product and process controls similar to reference product. Some aspects of the control strategy may rely on real time monitoring of CQA's which are determined through the implementation of process analytical technology (PAT). In the best scenario, process parameters maintain the control points flexibility/acceptability for the adjustments based on the outcomes of the real-time testing. The QA control strategy is tied closely with the risk assessment and the CQA's which are highly critical and considered to have a higher risk profile with respect to patient safety and effectiveness. The QA control strategy should take into account raw material quality as well, with a focus on design controls entry into the product life-cycle. Raw materials which are used in early process stages can be subsequently removed and generally pose a lower degree of risk to the intended user. As mentioned before, the goal of $\mathrm{QbD}$ is to utilize experience gained during development of the biosimilar product and the process to identify potential sources of variability. Once identified, mitigation strategies can be useful to reduce overall variability both in the particular product and its process. HACCP CCP's monitoring are useful to assess overall process performance, providing an evaluation of the total product life cycle. As mentioned before, continuous quality improvement is the ultimate goal of QbD.5-7

\section{Risk-Benefit Assessment:}

The developmental aspects of a novel biosimilar product requires, input $\mathrm{CMC}$, clinical pharmacology profiles, incorporation of regulatory essentials, and feasible marketing strategies. QbD concepts start with the projected benefits of the new biosimilar drug's clinical applications.1,2,4,7 This process is iterative based on the knowledge gained on the stereo-chemical aspects of clinical applications and drug product's dosing requirements; however, in the development of a biosimilar drug, many aspects of the new products are already considered in comparison to the FDA approved reference listed drug (RLD).2,3,7 The essential points in establishing biosimilarity is to demonstrate that the new product is the same in dosage strength, route of administration, and PK/PD profiles. Sponsors developing new biosimilar products, provide chemistry and biological properties of the drug molecule similar to reference products.3,5,7 Analytical similarity studies are the initial steps in the sequence of establishing products' similarities and serves feasibility for regulatory approaches to non-clinical and clinical studies. Ultimately, well-designed and well-managed studies will contribute to a successful biosimilar drug's end-development and premarket approval.4,5,9

\section{Summary:}

Biosimilar medicines are highly similar to FDA approved reference biologics. The sponsor's intended use claim plays an important role for use of biosimilar medicines in specialty therapy categories such as immunology, endocrinology, oncology. The new biosimilar drugs approved by FDA play a pivotal role in the clinical treatments of patients suffering from life threatening diseases such as cardiac myopathies, carcinoma, sarcoma, and lymphoma. The US biosimilar approval process requires a thorough characterization of the new biosimilars with clinically meaningful outcome. Sponsors of new biosimilar drugs follow the appropriate ICH guidelines in regard to clinical PK/PD, safety and efficacy studies. The FDA guidances for extrapolation and interchangeability state that data derived from clinical studies be adequate to demonstrate purity, potency, safety, and the intended clinical use of the new biosimilar drug similar to previously approved licensed biologics drugs. ${ }^{6-8}$

\section{Conclusion:}

This review article addresses quality system approaches to the development, GMPs, Quality Risk Management of new biosimilar biologic drugs presented in clinical applications. Emphasis is placed on HACCP-based Quality Risk Monitoring providing oversight and objective review of risk-benefit assessment that guides the clinical use of the new biosimilar drug product by providing patients organized data and appropriate labeling information in support of the new drug's intended clinical use. The sponsors of Biosimilar premarket applications must present analytical and biological characterization to demonstrate that a proposed biosimilar drug is highly similar to licensed reference product. The premarket application protocol requires a sponsor to describe the biosimilar drug's PK/PD clinical data comparing its safety, efficacy, and immunogenicity to that of the licensed reference drug. Discussions are presented on Design of studies and cGMP risk-based monitoring. A brief discussion is presented on risk-benefit assessment that guides the use of the new biosimilar drug product by providing clinically organized data and appropriate labeling information in conformance with the FDA's guidances on new drug approval for clinical applications.

\section{References:}

1. Aziz, KJ., Biosimilar drug development and approval process. J. Pharmacut Sci \& Emerging Drugs. 2019; 7(2):1-5

2. Aziz, KJ., Biosimilars: The Process and Quality System Approach to Clinical Applications. Drug Development \& Delivery. 2020; 20:5:1-7

3. Aziz, KJ., The FDAs New Drug Appeoval Process: Development and Premarket Applications. Drug Development \& Delivery. 2016; 3:50-56

4. Aziz, KJ., The FDAs New Drug Approval Process: Development and Clinical Applications. Drug Development \& Delivery. 2018; 4:1-10

5. Aziz, KJ., Pharmaceutical Manufacturing and Risk Management using Global HACCP Principles and Techniques. 2008, First and 4th Editions, Medical HACCP Alliance- Virginia Polytechnic Institute and State University, Blacksburg, VA

6. Lee, SL., et al., Modernizing Pharmaceutical Manufacturing from Batch to Continuous Production. J. Pharm Innovation. 2015; 10(3):191-199

7. U.S. Food and Drug Administration: Pharmaceutical cGMPs for the 21st Century- A Risk Based Approach. https://www.fda.gov/downloads/Drugs/Developmentapprov alprocess/manufacturing/questionsandanswerspractices gmpfordrugs/ucm176374.pdf.Published 2004.Accessed January 8, 2019

8. Tesser J., et al., Biosimilars and the Extrapolation of Indications for inflammatory conditions. Biologics; 2017; 11:5-11

9. Yu, LX., et al., Understanding Pharmaceutical Quality by Design. AAPS J.; 2014; 16(4):771-83

10. Singh SR, et l., Effect of Polysorbate 80 - Quality of Photostability of a monoclonal antibody. AAPS Pharm Sci Tech. 2012; 13(2):422-430 